\title{
Reproducible research and model validation: Are we ready for new standards?
}

\author{
Adin-Cristian Andrei, $\mathrm{PhD}$
}

From the Department of Surgery, Northwestern University Feinberg School of Medicine, Chicago, Ill.

Disclosures: Author has nothing to disclose with regard to commercial support.

Received for publication April 17, 2016; accepted for publication April 18, 2016; available ahead of print May $13,2016$.

Address for reprints: Adin-Cristian Andrei, PhD, Department of Surgery, Feinberg School of Medicine, 676 N St Clair St, Ste 1700, Chicago, IL (E-mail: a-andrei@northwestern.edu).

J Thorac Cardiovasc Surg 2016;152:356

0022-5223/\$36.00

Copyright (c) 2016 by The American Association for Thoracic Surgery

http://dx.doi.org/10.1016/j.jtcvs.2016.04.062

Hickey and Blackstone ${ }^{1}$ are to be congratulated for their excellent and very timely article on external validation for binary clinical risk prediction models. At a time when reproducible research is receiving increasing attention in the scientific community, it is critical that adequate efforts be made toward external model validation. ${ }^{2}$

Why is external model validation important? Because a good and useful model should be able to not only keep up with current data, but also improve as new data are being generated. As a musical instrument needs regular tuning to maintain its quality, a statistical model, even if built adequately at first, will be of limited use if incorrectly or insufficiently maintained. Population shifts or changes in operative techniques spurred by technological progress will affect the validity of any risk prediction model over time. In other words, once changes have occurred in the data-generating mechanism, the model needs to adapt to the new data reality and relearn the fundamental features of the mechanism. This could be an example of what Hickey and Blackstone adequately call a model "calibration shift." 1

External model validation is also important because as researchers we often attempt to conduct confirmatory analyses. It is rather common that certain variables, identified as risk factors by a research group, fail to show statistical significance on a different dataset. Why does this happen and what can be done to minimize the potential for such unsettling situations? Assuming that the data-generating mechanism is the same, one first needs to make sure that the model adequately captures the signal in the data, yet as little of the noise as possible. Resampling techniques are available to help achieve this goal. ${ }^{3}$ If the model captures too much of the noise in the data (a form of overfitting) then its features will be difficult to replicate externally for a simple reason: Noise does not replicate well. Commonly used statistical software provides ample options for model validation and it is up to us, as a scientific community, to

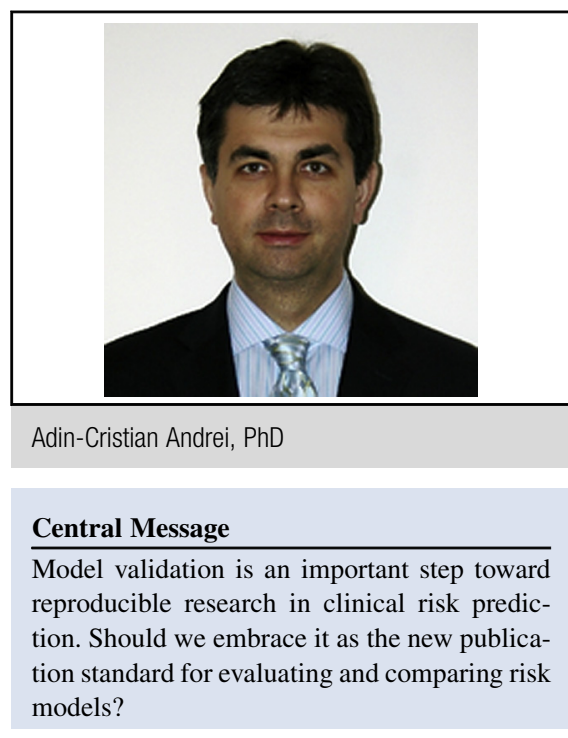

See Article page 351 .

uphold model validation, at least internally, as the new standard. Done properly, model validation will reduce the noise and improve consistency in findings across studies. In contrast, when the data-generating mechanism differs between the dataset used to fit the model and the external validation data, one should not expect external model validation to work well simply because the model has not been built to reflect the features of the validation data.

An important message that Hickey and Blackstone ${ }^{1}$ are communicating is that clinical risk prediction, and science in general, requires an increasingly sophisticated level of biostatistical knowledge and awareness of recent methodologic developments. Therefore, attracting top biostatistical talent remains a high priority. Continuing education courses at major society meetings focused on biostatistical aspects in general, and on model validation in particular, would also benefit those involved in clinical research.

\section{References}

1. Hickey GL, Blackstone EH. External model validation of binary clinical risk prediction models in cardiovascular and thoracic surgery. J Thorac Cardiovasc Surg. 2016;152:351-5.

2. Iqbal SA, Wallach JD, Khoury MJ, Schully SD, Ioannidis JP. Reproducible research practices and transparency across the biomedical literature. PLOS Biol. 2016;14:e1002333.

3. Steyerberg EW. Clinical prediction models: a practical approach to 244 development, validation, and updating. New York: Springer; 2009. 